REVIEW ARTICLE

\title{
Understanding Digital Filters in Polysomnography for Clinicians
}

\author{
Deepak Shrivastava, MD, FAASM, RPSGT; Amit Kalra, BS
}

Division of Sleep, Pulmonary and Critical Care, San Joaquin General Hospital and University of California, Davis Stockton, California, USA

DOI No:

Indian J Sleep Med 2013; 8.3, 111-115

$\mathrm{P}$ olysomnography (PSG) is becoming a routine procedure in the evaluation of sleep disordered breathing. There is not enough opportunity to learn technical aspects of PSG for clinical practitioners who are involved in the care and management of patients with sleep apnea. This review simplifies the concepts of signal processing and provides an easy to understand account of digital filters used in PSG.

This is important to understand a few basic concepts of electricity and current. Electric current is a flow of electrical charge that is carried by moving electrons. The current is measured in ampere. The direct current (DC) flows in one direction between positive and negative terminals. DC flows in a conductor like a wire, semiconductor and even in vacuum as in electron beams. In the Alternating Current (AC) the direction of the current reverses or alternates at a rate of 50 cycles per second (CPS) or $50 \mathrm{Hertz}(\mathrm{Hz})(60 \mathrm{CPC}$ or $\mathrm{Hz})$ in some countries).

\section{Impedance}

Impedance is opposition to alternating current (AC) flow, and it has two components, resistance and reactance. Resistance by itself is opposition to direct current (DC) flow, and in the context of impedance is a frequencyindependent opposition to AC current flow. The

\section{Correspondence Address}

Dr. Deepak Shrivastava, MD, FAASM, FCCP, FACP

Department of Internal Medicine

Division of Sleep, Pulmonary and Critical Care

San Joaquin General Hospital and University of

California, Davis

Stockton, California 95231, USA impedance is measured in Ohms (\&!). To understand impedance let us look at the analogy of water flowing through a pipe. The diameter of the pipe represents the impedance and the water flowing through it is the power. A larger diameter pipe will allow a larger amount of water to flow through it. But it will require the water pump to work very hard to maintain the water pressure. This would be considered the "low impedance" situation. Likewise, if the pipe were small in diameter it would allow less water to flow. This would be the "high impedance" situation as the smallness of the pipe is impeding or slowing the flow. That means low impedance equals large flow and high impedance equals slow or low flow. Voltage (water pressure) and current (water flow) together create power. If the size of the pipe changes to larger, the voltage would not change but the current would drop! The amplifier (water pump) has to work harder to provide more current to maintain flow. The higher demand on the amplifier could cause its malfunction. Therefore the impedance has to be in a certain optimal range to obtain the optimal signal quality.

Very high or very low impedance is not desirable. Very low impedance acts like a shunt between the recording electrodes and effectively short-circuits the EEG potential differences. It is practically impossible to reduce electrode impedance to less than a few hundred Ohms without creating an abnormal pathway of conduction between the electrodes across the scalp. An electrode showing very low impedance may be making contact with another electrode because of an excess of electrolyte jelly or paste, or because of saline or sweat forming a conductive bridge between the electrodes on the scalp. Such an electrode should be inspected, cleaned, and reapplied or exchanged if necessary. 
Very high impedance is undesirable mainly because connecting an electrode of very high impedance and one of lower impedance to the input of a differential amplifier causes an imbalance which favors the recording of 50 $\mathrm{Hz}$ interference $(60 \mathrm{~Hz}$ in some countries). Electrodes showing high impedance readings should be checked for good mechanical and electrical contact and the junctions of the lead wire with the metal disc or cup and with the plug terminal should be inspected for possible breaks.

\section{Amplifier}

This is an electronic devise that increases the power of a signal. It takes the energy from a power supply and controls the signal output to match the signal input without changing its original shape. Amplifier only increases signal amplitude. In other words an amplifier modulates the output of the power supply. Amplifiers are equipped with the filters both for the low and high frequencies. The conceptual understanding of the digitization of the analog signal is important to fully comprehend the function of the filters. The analog or continuous signal from the patient is converted in to numeric digital form by an analog to digital converter. The number of sampled intervals obtained in the span of one second is defined as the sampling rate. In the digital recordings the sampling rate and intervals are important as it may work as a filter. For example, if a sample is collected every fifth of a second, a sleep spindle would be missed; or in parasomnias when seizure is a concern the recommended $100 \mathrm{~Hz}$ sampling rate is inadequate. Spikes occurring in the $100 \mathrm{~Hz}$ range would be missed $^{1}$

\section{Differential amplifier}

An electronic amplifier, amplifier, or in popular terms "amp" is an electronic device that that increases the power of a signal. Differential amplifier draws energy from a power supply and controls the output to match the input signal shape but with larger amplitude. It is important to understand that differential amplifier does not amplify the input signal but instead it amplifies the difference between two inputs terminals. In other words, the output of a differential amplifier reflects the voltage difference between the two inputs (input 1 and input 2).Another critical concept is that of the polarity convention. It dictates thatif the difference is a negative value or input 1 is relatively more negative than input 2 the deflection of the EEG activity goes up. Similarly, if the difference between the input 1 and input 2 is a positive value, then deflection goes down. If there is no difference between input 1 and input 2 there will be no deflection in voltage.

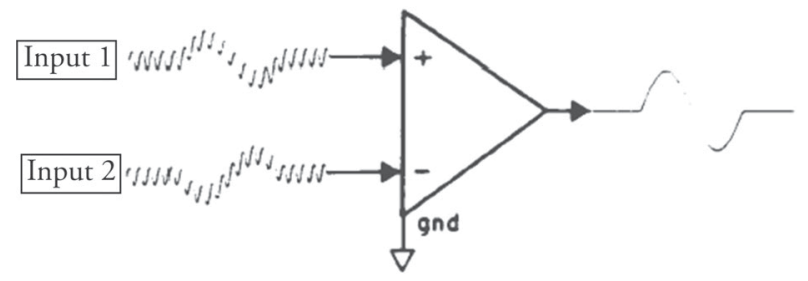

Differential amplification: Input signals 1 and 2 above have two frequencies. The high-frequency signal is common mode (same amplitude and phase at the two inputs) and is rejected. In contrast, the low-frequency signal is the opposite phase and is enhanced. Modified and referenced per instructions: American Speech Language-Hearing Association, (1987)

The amplifier gain is the ratio of the output signal to the input signal. Optimum signal quality and adequate voltage for continued signal processing is provided by proper gain. Other parameters like range of the analog to digital convertor and sampling rate influence the signal quality. Differential amplifier should be distorting effects of the electrical noise; amplifiers have a high common mode rejection ratio of at least $100 \mathrm{~dB}$ and a high input impedance of at least $100 \mathrm{M}$ Ohms. The common mode rejection ratio is the ratio of the gain of the desired signal (differential mode) over the original input signal between the inputs and ground (common mode). The amplifiers are not perfectly balanced and the degree of the "imperfection" is called the common mode rejection ratio.

A basic engineering principle known as Nyquist law states that when a waveform has to be reconstructed, no signal with a frequency exceeding half the sampling rate is allowed at the input of the analog-digital converter. Above the Nyquist frequency, the frequency components are impossible to detect. In fact, they appear to have a frequency less than the Nyquist frequency. For example, when sampling at $200 \mathrm{~Hz}$, a $110 \mathrm{~Hz}$ wave is seen as 90 $\mathrm{Hz}$ wave, a $125 \mathrm{~Hz}$ wave as a $75 \mathrm{~Hz}$ wave and a $199 \mathrm{~Hz}$ wave as $1 \mathrm{~Hz}$ wave. This misreading of waves is called aliasing. Anti-aliasing analog filters provide this function. For a sampling rate of $200 \mathrm{~Hz}$, the anti-aliasing filters will remove everything higher than $100 \mathrm{~Hz}$ (any frequency higher than the one half of the digitization rate) prior to digitization $^{2}$. In order to display the waveforms similar 
to the "real paper recording", the sampling tare should be at least four to five times the maximum desired visible frequency. For example, to identify a $30 \mathrm{~Hz}$ waveform the desired minimum sampling rate should be at least $150 \mathrm{~Hz}^{3}$.

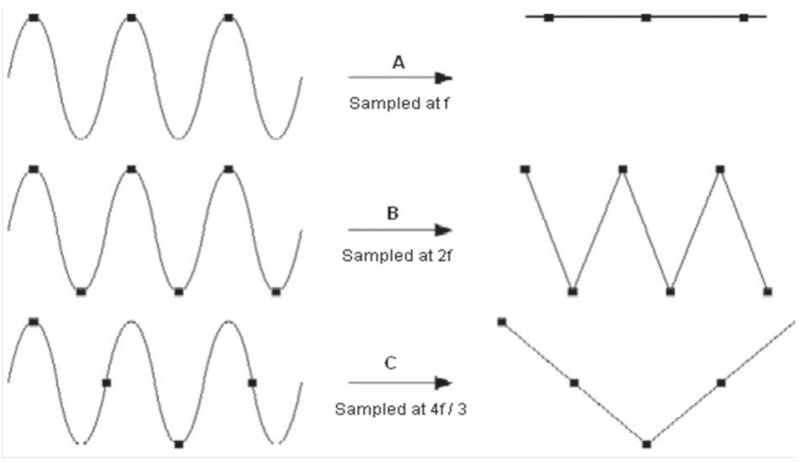

The figure above shows the effects of various sampling rates. In $A$, the sine wave frequency is sampled at the same frequency. The reconstructed wave form appears as an alias. When the sampling rate is increased to 2 times in $B$, the digitized waveform has correct frequency (same number of cycles) but appear as a triangular waveform. This frequency is equal to the Nyquist frequency. In $C$, sampling rate is well above the frequency itself; this sampling rate produces an alias waveform of incorrect frequency and shape.

Filters provide a method of isolating specific frequency bandwidths. In polysomnography the electroencephalogram (EEG) is recorded within a bandwidth of approximately 0.5 to $25 \mathrm{~Hz}$, representing the frequency range necessary to identify stages of sleep and wakefulness. The low and high filters are set accordingly. The unwanted slow frequencies like the skin or fast frequencies from the muscle activity can be removed $^{4}$

The conventional analog filters use resistors and capacitors to attenuate undesired frequencies, while digital filters use software based algorithms to accomplish the same. The amplified signal is converted in to digital form before being presented for filtering. Digital filters provide great flexibility and raw data can be collected without the application of any filter. Data display during the data collection or during playback can selectively filtered without altering the original recorded data ${ }^{5}$.

In general, filters are applied to prevent aliasing and to eliminate unwanted low frequency signals that are not of biological origin or interest. Filtering alters the amplitude and the shape of the waveforms. Filters are used to allow certain frequencies to pass unchanged and block, attenuate or filter others thereby enhance the quality of the desired signal. Filters remove unwanted frequencies and make the data recordings clean and interpretable. Frequencies are described in terms of cycles per second (CPC) or hertz (Hz). The low-pass filter (LF) allows low frequencies go through and stops and dampens the higher frequencies. The filter may have a different damping response to different frequency. The high-pass filter (HF), on the other hand allows the high frequency signal to pass and be displayed. It stops and dampens the low frequency signals. The terminology is somewhat confusing in the literature as many authors refer Low pass filter (LF) as High-frequency filter or high cut-filter suggesting that high frequencies are removed while low frequencies are allowed to pass. Similarly, the High pass filter (HF) is referred as Low-frequency filter or low cut filter suggesting lower frequencies are stopped and high frequencies are allowed to pass. Notch filter remove the frequencies due to electrical interference generally at 50 $\mathrm{Hz}$ or $60 \mathrm{~Hz}$ as the case may be.

Further, it is important to understand the concepts of time-constant. This is the time required for a square wave voltage to decay to $63 \%$ of its peak amplitude. Time constant describes the effects of a high-pass filter on the square-wave pulse $e^{6}$. In electrical terms the time constant is equal to the product of the resistance and the capacitance ( $\mathrm{TC}=\mathrm{RXC}$ ). There is an inverse relationship between the time constant and the high-pass filter. The higher the high-pass filter, the shorter the time constant and the lower the high-pass filter, the longer the time constant. Therefore, by setting a longer time constant for an amplifier, slower frequency waveforms are amplified without filtering. Filters are described in terms of their frequency response curves.

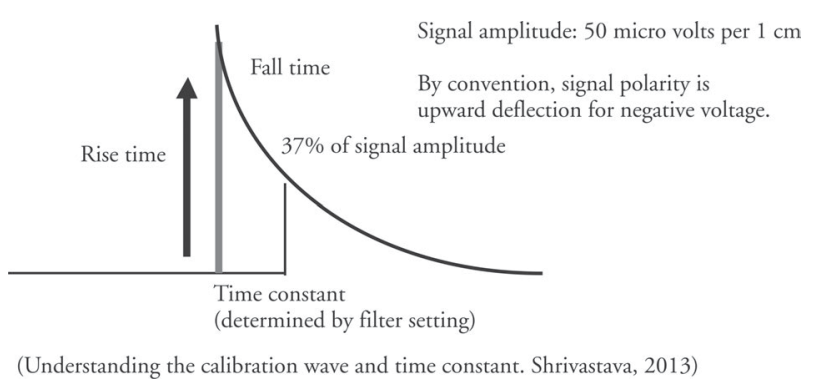

\section{Low-Pass Filters}

Low pass filter (High frequency filter) remove high frequency signal and allow the lower frequencies to pass through. When a cut-off frequency is specified, then the amplitude of any higher frequencies signal is reduced. 
The reduction rate of the amplitude or "roll-off" is steeper in low-pass filters.

The advantage of having a steeper "roll-off" is that it allows analog filtering prior to digitalization. In turn, a lower sampling rate becomes acceptable for analog to digital converter.

\section{High-Pass Filters}

High-pass filter (Low frequency filter) removes slower frequencies like respiratory artifact. When a cut-off frequency is specified the amplitude of that frequency is reduced by $20 \%$ to $30 \%$. The gradually slowing frequencies are reduced severely in a "roll-off'

\section{Notch Filters}

Notch filters remove electrical interference generated by other electrical devices that use alternating current (AC), typically at $60 \mathrm{~Hz}$ frequency. Notch filters reduce $60 \mathrm{~Hz}$ frequency severely but may not remove it completely ${ }^{7}$. The appearance of a $60 \mathrm{~Hz}$ artifact may indicate unequal impedance between electrodes like poor skin contact or sweating. Other common causes include defective lead wires, inadequate grounding or nearby electrical equipment. Such problem should corrected first before considering the use of a notch filter. The use of notch filter may obscure a physiologic signal of interest like spike waves.

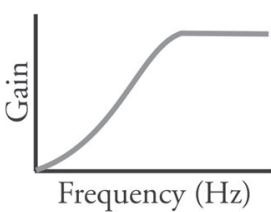

Figure A

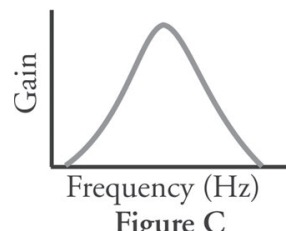

Figure $\mathrm{C}$

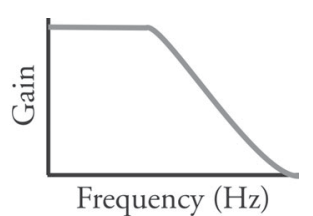
Figure B

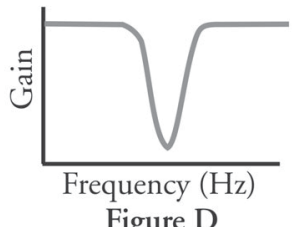

The figures above show Frequency Response Curves using different types of filters. Figure A shows a High-Pass Filter Curve, with frequencies below the cutoff frequency gradually attenuated. Figure $B$ shows a Low-Pass Filter Curve, with frequencies above the cutoff gradually attenuated. Figure C shows a Band-Pass Filter Curve, with frequencies both above and below the cutoff frequency attenuated. Figure D shows a Notch Filter Curve, with frequencies within a certain bandwidth attenuated. (A. Kalra, 2013)
Following example recordings are obtained from Alice Sleepware by Respironics version 2.8 (Alice 5 software) to illustrate the visual impact on the amplitude of the EEG waves in C3M2 and C4M1 derivations. Other available digital sleep recording systems demonstrate similar capabilities to digitally improve data manipulation and quality without permanent alteration of the original data.

\section{PANEL A}

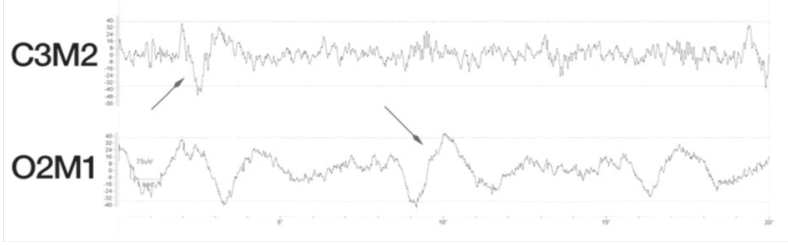

PANEL B

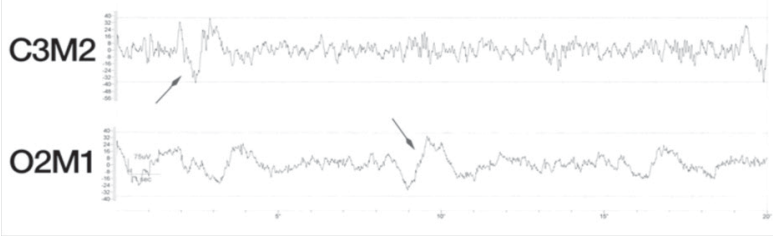

PANEL C

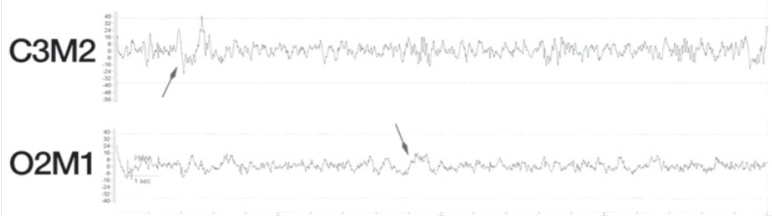

Panel A, shows two EEG waves processed digitally using a HighPass Frequency Filter of $0.3 \mathrm{~Hz}$. Notice the amplitudes of the artifact waves indicated by the red arrows. Panel B again shows the same segment of the same EEG waves, but is now digitally filtering out all frequencies less than $0.5 \mathrm{~Hz}$. Moderate attenuation of the marked waves is evident. In Panel $C$, the High Pass Frequency filter is set to $1.0 \mathrm{~Hz}$. using this filter setting; the wave amplitudes are severely attenuated. These digital filter manipulations can allow for the exclusion of unnecessary EEG waves from the recording, allowing for only pertinent data to be presented and evaluated. (A. Kalra, 2013) 
American Academy of Sleep Medicine (AASM) recommends the following filter settings from the AASM manual ${ }^{10}$.

\begin{tabular}{|l|l|l|}
\hline $\begin{array}{l}\text { Filter settings } \\
\text { for recording }\end{array}$ & $\begin{array}{l}\text { Low frequency } \\
\text { filter }\end{array}$ & $\begin{array}{l}\text { High frequency } \\
\text { filter }\end{array}$ \\
\hline $\begin{array}{l}\text { Electroencephalogram } \\
\text { (EEG) }\end{array}$ & $0.3 \mathrm{~Hz}$ & $35 \mathrm{~Hz}$ \\
\hline $\begin{array}{l}\text { Electro-occulogram } \\
\text { (EOG) }\end{array}$ & $0.3 \mathrm{~Hz}$ & $35 \mathrm{~Hz}$ \\
\hline $\begin{array}{l}\text { Electromyogram } \\
\text { (EMG) }\end{array}$ & $10 \mathrm{~Hz}$ & $100 \mathrm{~Hz}$ \\
\hline $\begin{array}{l}\text { Electrocardiogram } \\
\text { (EKG) }\end{array}$ & $0.3 \mathrm{~Hz}$ & $70 \mathrm{~Hz}$ \\
\hline Respiration & $0.1 \mathrm{~Hz}$ & $15 \mathrm{~Hz}$ \\
\hline Snoring & $10 \mathrm{~Hz}$ & $100 \mathrm{~Hz}$ \\
\hline
\end{tabular}

Understanding the science of generation and recording of the electrophysiological signal is of critical importance. The recorded data may be affected by the contaminated signal and may significantly impact the interpretation of data and subsequent clinical decision making. A thorough review of the technical aspects of data signal processing may improve ability to recognize the erroneous recording, correcting the problem and finally recording a high quality polysomnogram.

\section{References and recommended reading}

1. SP Patil, ACCP Sleep Medicine Board Review: 4th Edition

2. CurrProtocNeurosci. 2009

3. Analog to digital conversion: Journal of Clinical Sleep Medicine 2007; 3,(2)

4. Mary Carskadon, Sleep medicine 2002; 608-611

5. Fundamentals of Sleep Technology, by Teofilo L. Lee-Chiong Jr., NicButkov, Mary Jones-Parker, Lee J. Brooks

6. Fisch BJ. Digital and analog EEG instruments: parts and functions. In: Fisch BJ, ed. Fisch and Spehlmann's EEG primer: basic principles of digitaland analog EEG. Amsterdam, The Netherlands: Elsevier, 1999; 35-72

7. Thomas SJ. Basic principles of polysomnography including electrical concepts. Respir Care Clin North Am 2005; $11: 587-59$

8. Penzel $\mathbf{T}$; Hirshkowitz $M$; Harsh J et al. Digital analysis andtechnical specifications. J Clin Sleep Med 2007; 3(2): 109-120

9. Hirshkowitz $\mathbf{M}$; Sleep medicine reviews, 2000; 4:169179

10. Berry RB, Brooks R, Gamaldo CE, Harding SM, Lloyd RM, Marcus CL and Vaughn BV for the American Academy of Sleep Medicine. The AASM Manual for the Scoring of Sleep and Associated Events: Rules, Terminology and Technical Specifications, Version 2.0.2. www.aasmnet.org, Darien, Illinois: American Academy of Sleep Medicine, 2013. 